\title{
Quadratic Tensor Anisotropy Measures for Reliable Curvilinear Pattern Detection
}

\author{
Mohsin Challoob and Yongsheng Gao \\ School of Engineering, Griffith University, Brisbane, Australia \\ mohsin. challoobegriffithuni.edu.au, \\ yongsheng.gao@griffith.edu.au
}

\begin{abstract}
A wide range of applications needs the analysis of biomedical images as a fundamental task to extract meaningful information and allow high throughput measurements. A new method for the detection of curve-like structures in biomedical images is presented by exploiting local phase vector and the structural anisotropy information at various directions. We introduce an oriented gaussian derivative quadrature filter not only for estimating the local phase vectors, which include line features, but also for its immunity to inhomogeneous intensity and its capability to enhance curved structures having various diameters, leading to more reliable hessian analysis. A novel measure functionbased hessian tensor is proposed to detect curvilinear patterns by incorporating the anisotropic indices (coherence and linearity) of curved features, producing a uniform and strong response. Over multiple orientations, the responses are maximized to achieve a rotationally invariant response, and to detect target structures with different widths and illuminations. The evaluation of the proposed method on the extraction of retinal vessels and leaf venation patterns exhibits its superior performance against state-of-the-art methods.
\end{abstract}

Keywords: Curvilinear detection. Quadratic tensor anisotropy. Measure indices

\section{Introduction}

The analysis of biomedical imaging data plays a signification role in health care purposes and life sciences. In particular, the accurate enhancement, detection, quantification, and modelling of curve-like patterns is an essential step in bioimage informatics and medical quantification. For example, retinal blood vessels provide clinicians with information to diagnose, screen, and evaluate a wide range of pathologies such as diabetes and arteriosclerosis, and the automatic extraction of leaf veins is desirable for the classification of plant species [1-4]. However, the detection of curvilinear structures in biomedical images is a challenging task due to several difficulties including the changes in the intensity, the variability of features (width, length, tortuosity), inhomogeneous background, noise, and poor contrast between curved patterns and the background. A number of automatic detection approaches has been introduced to extract curve-like patterns from biomedical images, but these methods suffer from many weaknesses. A phase congruency tensor (PCT) [2], which was introduced for 
detecting curvilinear structures in biomedical images, is sensitive to noise and unable to detect curved structures with different diameters, where the phase congruency cannot distinguish between lines, edges and mach bands and fuses them together. The morphological approaches $[4,6]$ apply an overly long line-shape structuring element rotated at several directions. This results in a difficulty in detecting high tortuosity patterns, produces a non-uniform response, and increases the sensitivity to background noises. An isotropic nonlinear filtering approach [5] applies circular masks to detect lines which are narrower than maximum width parameter and there is no bandpass filtering capability, leading to the deficiencies of the susceptibility to intensity inhomogeneity, structure width size, and non-uniform response across different curved morphologies. The studies [7] and [8] use hessian matrix to measure the response of vesselness and neuriteness, respectively, where this matrix is computed with the second order derivatives of gaussian. These hessian-based methods tend to suppress junctions and rounded structures, causing the discontinuity of curved network. The weaknesses of state-of-the-art methods are summarized as follows:

- Difficulty in detecting curvilinear structures with different diameters (thick, median, fine) when presenting together.

- Non-uniform response across various structures due to the lack of dealing with changes in illumination.

- Loss of the curved tree connectivity because of the suppression of junctions and rounded structures.

- Susceptibility to noise, particularly in regions with uniform intensity.

This paper introduces a new method for the detection of curve-like structures, which overcomes the mentioned deficiencies of the state-of-the-art algorithms. The proposed method incorporates the local properties of line-like features derived from the local phase vector-based gaussian derivative quadrature (GDQ) filter, with the coherence and linear anisotropy information of curvilinear patterns acquired from hessian tensor. Due to the fact of relating structural information to a given local orientation and to deal with the presence of various structure diameters together, a set of GDQ filters are used at multiple directions to estimate the local phase vectors. Further, the local phase based-GDQ filter has an inherent feature invariant to changes in intensity, and can discriminate between lines and edges, which in turn contributes to achieving robust hessian analysis. Then, we present a new measure function-based hessian tensor by quantifying the linearity of local anisotropic intensity and the local coherence of target structure to yield a strong and uniform response for various structure widths. The proposed function automatically measures the response without any threshold parameter controlling the sensitivity of the function and indices, except the scale range determining the size of target structure. To make sure that curvilinear structures with different diameters are captured, the responses of the measure function are maximized over multiple angles to produce a response at each pixel. The proposed method scheme (decomposition-filtering-recombination) preserves network connectivity while retaining thin and small curved patterns. The assessment that is conducted on retinal vessels and leaf veins shows the effectiveness of the proposed method, yielding excellent results which outperform the previous methods. 
The reminder of this paper is organized as follows. In Section 2, the proposed method is presented, where the GDQ deriving the local phase and anisotropic indicesbased the proposed measure function are explained in detail. Section 3 shows the experiment results including the quantitative and qualitative assessment and the comparison with existing methods, whereas the discussion and conclusion are stated in Section 4.

\section{Methodology}

In this section, a novel method for the detection of curvilinear structures in 2D biomedical images is proposed. The laminar flow within biological structures such as vessels and veins lead to flow velocity variations, which in turn cause the changes in illumination and non-uniformity of intensity across various curvilinear structures running at different widths and orientations. To deal with variations in intensity and scales, and suppression of undesired structures such as noise, we firstly introduce an oriented gaussian derivative quadrature (GDQ) filter to estimate local phase vector at various angles. Then, a novel measure function for the detection of curved structures is presented based hessian tensor by quantifying the coherence and linearity indices of local anisotropy information to produce a uniform response across various curved structures while being insensitive to noise regions with uniform intensity.

\subsection{Local Phase-based GDQ Filter}

Local phase is a key local feature for determining the structural information (lines, edges) of an image and can be defined in 3D space under the concepts of monogenic signal, which assumes that signal comprises of frequencies with bandlimited [9]. We introduce a gaussian derivative quadrature (GDQ) filter to estimate local phase vector. The phase map includes the location and orientation of the image features compared to amplitude carrying their intensity only, see Figure 1 (first row), where the contour plotting is shown for the magnitude, real part and imaginary part of local phase of an example image. Also, we use the phase-based GDQ filter rather than other intensitybased filters as it is insensitive to changes in illumination (zero DC component), and can exploit line-like patterns, which can be distinguished independently from other image features. According to [9], the GDQ filter has an advantage of allowing a continuous bandwidth with a maximum bandwidth of (2.59) octaves, having less aliasing than other quadrature filters such as log-Gabor and difference of gaussian which are with extended tail. By utilizing the derivative property of Fourier transform, the GDQ filter is defined in frequency domain as follows:

$$
G_{d}(w)=\left\{\begin{array}{ll}
n_{c} w^{a} \exp \left[-\sigma^{2} w^{2}\right] & \text { if } w \geq 0 ; \\
0, & \text { otherwise }
\end{array} \quad \text { where, } a \in \mathbb{R} \text { and } a \geq 1\right.
$$




$$
n_{c}=2 \frac{\sqrt{\pi} \sigma^{\left(a+\frac{1}{2}\right)}}{\sqrt{\Gamma\left(a+\frac{1}{2}\right)}}
$$

where, $n_{c}$ represents the normalized constant value, $\Gamma$ is the gamma function, and the peak tuning frequency is given by $\left(\frac{\sqrt{a}}{\sigma}\right)$. The local phase-based the GDQ filter is estimated as a vector response and is defined as $Q^{\theta}(x)$ at each point $x$ of the image $I$ as follows:

$$
Q^{\theta}(x)=E^{\theta}(x)+i O^{\theta}(x), \text { where } i=\sqrt{-1}
$$

The $E^{\theta}(x)$ and $O^{\theta}(x)$ denote the even-symmetric and odd-symmetric components (real and imaginary), respectively, at an orientation $\theta$. In practice, various angles are required to capture curvilinear structures distributed at different diameters and directions and therefore an oriented GDQ filter is introduced to derive a set of local phase vectors, where the curvilinear network is decomposed into several sub-directional structures. The filter response is dominantly real for lines as illustrated in Figure 1second row, where $E^{\theta}$ yields maximal response, while $O^{\theta}$ has almost zero response. In contrast, $O^{\theta}$ attains high response at edges whereas $E^{\theta}$ is almost zero (see Figure 1third row). Hence, we determine line-like structures from real part only, which is obtained as:

$$
E^{\theta}(x)=\operatorname{real}\left(F^{-1}\left(G_{d}^{\theta}(w) * F(I(x))\right)\right)
$$

where $F$ and $F^{-1}$ represents forward and inverse Fourier transforms, respectively.

\subsection{Anisotropic Indices-based Measure Function}

The line-like structures derived from the symmetric even (real) part has an anisotropic intensity. We propose a novel measure function-based hessian tensor not only to consider the degree of anisotropy, but also to produce a uniform and strong response for different structure diameters while being more robust against background noise. The proposed function utilizes the linear anisotropy index, which has been reviewed in [10], and the coherence measure used in [11]. Hessian tensor is performed on a gaussian scale space of the $E^{\theta}(x)$ at scale $s$, and is computed as a $2 x 2$ matrix $\left(H^{\theta}(x, s)\right)$, where eigenvalues $\left(\lambda_{i}\right)$ of the tensor are sorted based on their magnitudes. To capture anisotropic behavior, we evaluate the linearity of local anisotropic intensity and the local coherence of target structure. The linearity of anisotropy $\left(L_{a}\right)$ quantifies the variation of eigenvalues across various structures, where the change of anisotropy along the curved structures is measured. The coherence index ( $\mathrm{Coh}$ ) provides the actual amount of local anisotropy information. 


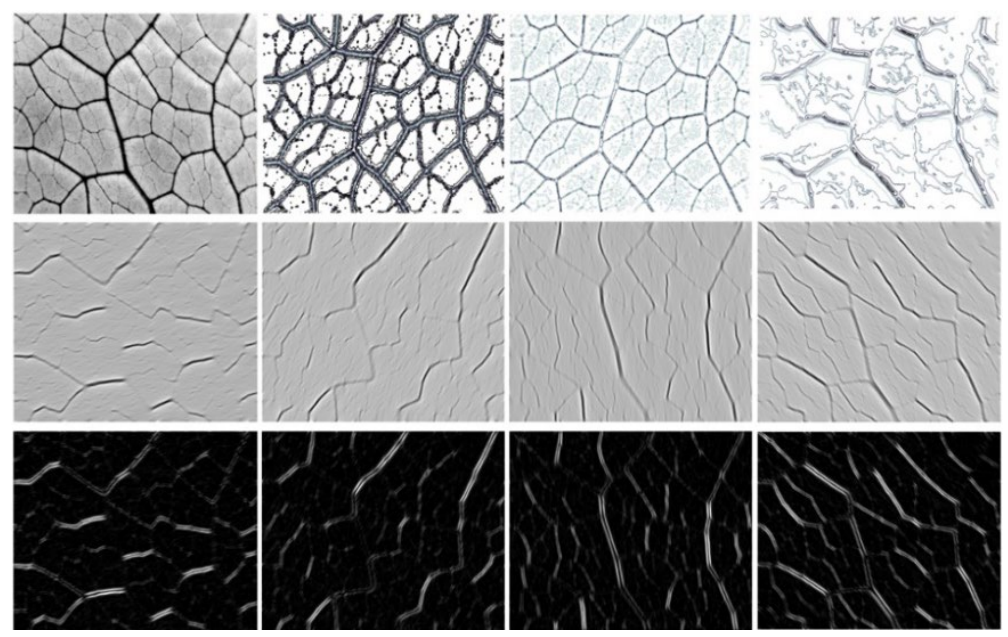

Fig. 1. First row: contour plotting for magnitude, real and imaginary of a leaf image taken from [2], respectively. Second and third rows: real and imaginary parts of local phase at multiple orientations, respectively.

The $L_{a}$ and $C o h$ indices are defined as:

$$
L_{a}=\frac{\lambda_{2}-\lambda_{1}}{\lambda_{1}+2 \lambda_{2}}, \quad \operatorname{Coh}=\left(\lambda_{2}-\lambda_{1}\right)^{2}
$$

These quantities are large for line-like structures while they tend to zero for isotropic structures (homogenous regions), where the greater local anisotropic intensity, the greater the difference between eigenvalues. Then, the proposed measure function $\left(V_{s}^{\theta}\right)$ is introduced as:

$$
V_{s}^{\theta}=\operatorname{Coh} \exp \left(-S_{t}\left(\frac{\lambda_{1}}{\lambda_{2}} L_{a}\right)^{2}\right)
$$

where $S_{t}=\sqrt{\lambda_{1}{ }^{2}+\lambda_{2}{ }^{2}}$ refers to the second order factor of structuredness. To produce invariant response to size and orientation, the final response $F_{v}(x)$ is obtained by maximizing the measure function $\left(V_{s}^{\theta}\right)$, at each pixel $x$, over a range of scales $(s)$, and a set of angles $(\Theta)$, as follows:

$$
F_{v}(x)=\left\{\max _{\theta \in \Theta}\left\{\max _{s_{\min } \leq s \leq s_{\max }}\left(V_{s}^{\theta}\left[\text { eig } H^{\theta}(x, s)\right]\right)\right\}\right\}
$$

where the range of scales $(s)$ determines the expected minimal and maximal size of curvilinear structures of interest. 


\section{Experimental Results}

The evaluation of the proposed detection method is conducted on fundus imaging (DRIVE dataset ${ }^{1}$ and STARE dataset ${ }^{2}$ ), leaf image dataset ${ }^{3}$, and sub images obtained from mouse retina ${ }^{4}$. Regarding the DRIVE and STARE datasets, the green band is only considered for its better contrast then prepressed using the morphologically approach in [12] for further enhancement. Figure 2 shows the detection results on these datasets, where the scale range $s=(0.2: 0.2: 5)$ is set for the DRIVE while $s$ $=(0.5: 0.5: 4)$ for the STARE, and $\Theta=\{\pi, \pi / 18,2 \pi / 18,3 \pi / 18, \ldots 17 \pi / 18\}$ is for both datasets. The venation patterns are extracted from leaf images, as illustrated Figure 3, under consideration $s=(0.5: 0.5: 3)$, and $\Theta=\{\pi, \pi / 12,2 \pi / 12,3 \pi /$ $12, \ldots 11 \pi / 12\}$. The extraction of vessel trees from sub-images of mouse retina is demonstrated in Figure 4, and is carried out with $\Theta=\{\pi, \pi / 12,2 \pi / 12,3 \pi /$ $12, \ldots 11 \pi / 12\}$, and $s=(0.1: 0.1: 2.2)$ for the first sub-image whereas $s=(0.5: 0.5: 5)$ for the others.

The performance of the proposed method on the DRIVE and STARE databases is quantitively assessed using three common metrics: accuracy (ACC), specificity (SP) and sensitivity (SE). The obtained results are compared with eleven state-of-the-art algorithms, see Table 1, where local entropy method is used for thresholding the final response of our method. For the DRIVE dataset, the results are calculated using the test set (20 images) with ground truth A (1st_manual). For the STARE database (20 images), the manual segmentations by the first observer are used as ground truth. The results of the algorithm [15] on both fundus imaging databases are obtained from the paper [18], while the rest are taken from published results in their original studies. As illustrated in Table 1, the proposed detection method performs best on the DRIVE against the supervised and unsupervised algorithms by producing the highest accuracy over other methods, higher sensitivity except the method in [16] and competitive specificity to others. On the STARE, the proposed method achieves a higher accuracy except the methods [17], [19] and [20], and a higher sensitivity excluding the methods [17] and [19].

The qualitative comparison is also carried out using randomly chosen retinal and leaf images (see Figures 5 and 6) with seven state-of-the-art detection algorithms: phase congruency tensor-vesselness (PCT-V) [2], phase congruency tensor-neurite (PCT-N) [2], multiscale bowler-hat transform (MBT) [4], isotropic nonlinear filtering (ISNF) [5], mathematical morphology with curvature evaluation (MCE) [6], vesselness detector (VD) [7], and neurite detector (ND) [8]. To have fair comparison, their parameters are optimized to produce best results. As shown in Figure 5, the ISNF and VD can only detect vessels with large diameter, missing small ones, and produce non-

\footnotetext{
1 https://www.isi.uu.nl/Research/Databases/DRIVE/download.php

2 http://cecas.clemson.edu/ ahoover/stare/

3 http://www.imageprocessingplace.com/root_files_V3/image_databases.htm, (developed by

V. Waghmare)

4 https://bisque.ece.ucsb.edu/client_service/
} 

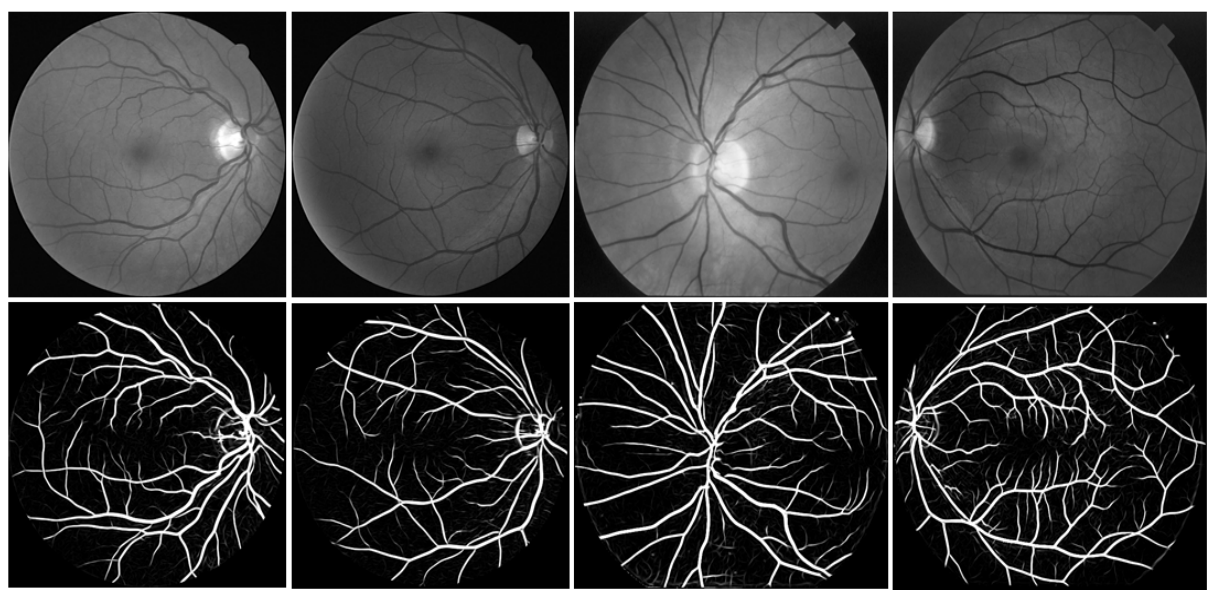

Fig. 2. First row: original images (the first two images from DRIVE while others from STARE) . Second row: detection results by our method.
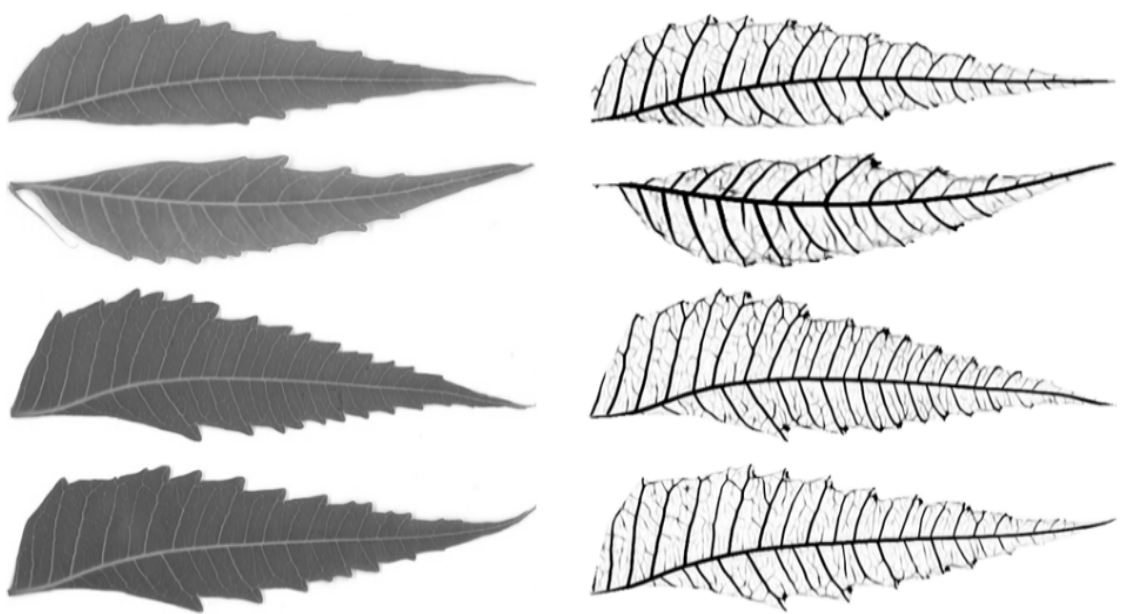

Fig. 3. First column: original leaf images. Second column: detection results by our method.

uniform response, while the PCT-V is susceptible to noise, especially in regions with uniform intensity and to light reflection running at the center of wide-diameter pattern. The methods MBT and MCE enhance background regions, which causes a challenge into distinguishing vessel tree, whereas the ND and PCT-N clearly produce false detection in junctions and crossings, losing the connectivity of the tree. In Figure 6 , the proposed method produces superior response on the detection of venation patterns, outperforming the other algorithms which fail into extracting vein network from leaf images. The methods PCT-V, MBT and ND enhance the background artifacts and produces noisy response. The methods ISNF, VD and ND miss the veins with 
fine diameters and produces discontinuous venation tree. The PCT-N and MCE yield very weak response to extract venation patterns as the PCT-N is sensitive to low intensity veins while the MCE is susceptible the intensity inhomogeneity, enhancing background regions with venation tree.
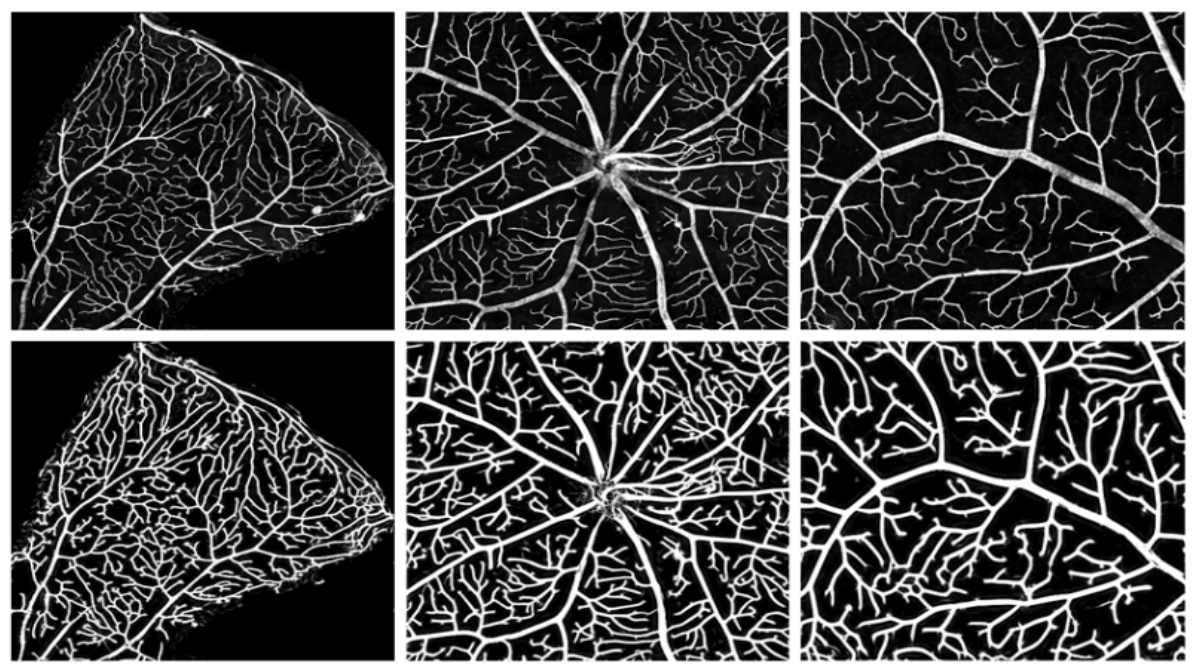

Fig. 4. First row: sub-images from mouse retina. Second row: detection results by our method.

Table 1. Performance comparison of proposed method with benchmark algorithms on DRIVE and STARE databases.

\begin{tabular}{l|ccc|ccc}
\hline Database & \multicolumn{3}{|c|}{ DRIVE } & \multicolumn{3}{c}{ STARE } \\
\hline \hline Method Type & ACC & SP & SE & ACC & SP & SE \\
\hline Supervised & & & & & & \\
\hline Marín [13] & 0.945 & 0.980 & 0.706 & 0.952 & 0.981 & 0.694 \\
\hline You [14] & 0.943 & 0.975 & 0.741 & 0.949 & 0.975 & 0.726 \\
\hline Soares [15] & 0.944 & 0.976 & 0.723 & 0.948 & 0.973 & 0.710 \\
\hline Lázár [16] & 0.945 & 0.972 & 0.764 & 0.949 & 0.975 & 0.724 \\
\hline Cheng [17] & 0.947 & 0.979 & 0.725 & 0.963 & 0.984 & 0.781 \\
\hline \hline Unsupervised & & & & & & \\
\hline Zhao [18] & 0.947 & 0.978 & 0.735 & 0.950 & 0.976 & 0.718 \\
\hline Zhao [19] & 0.954 & 0.982 & 0.742 & 0.956 & 0.978 & 0.780 \\
\hline Lam [20] & 0.947 & - & - & 0.956 & - & - \\
\hline Nguyen [21] & 0.940 & - & - & 0.932 & - & - \\
\hline Fraz [22] & 0.943 & 0.976 & 0.715 & 0.944 & 0.968 & 0.731 \\
\hline Zhang [23] & 0.938 & 0.972 & 0.712 & 0.948 & 0.975 & 0.717 \\
\hline Proposed Method & $\mathbf{0 . 9 5 6}$ & $\mathbf{0 . 9 7 6}$ & $\mathbf{0 . 7 4 7}$ & $\mathbf{0 . 9 5 4}$ & $\mathbf{0 . 9 7 0}$ & $\mathbf{0 . 7 6 0}$ \\
\hline
\end{tabular}


Further, we closely examined the response of the proposed method, in comparison with other algorithms, in weak contrast, junctions and crossings, and central light reflex, as exhibited in Figure 7. Based on the obtained results, the proposed method shows its superior to: I) detect low contrast pattern in inhomogeneous region as compared to the methods PCT-V and ISNF; II) produce stronger response at bifurcations than VD and ND methods; III) be more robust when presenting the central light than MBT and ISNF; IV) attain a more uniform response across various structure widths than other methods.

\section{Discussion and Conclusion}

The detection of curvilinear patterns is still challenging for existing methods due to variations in intensity, highly curved structures, inhomogeneous background, and variability of pattern diameters. In this paper, we exploit the key advantage of the local phase-GDQ filter, which includes the intrinsic information of line features that are invariant to changes in illumination as a way to handling the inhomogeneity of intensity, whereas the use of the GDQ filter at several orientations deals with various structure widths and directions. Also, the separation between lines and edges by the GDQ increases the robustness of the detection and avoids the duplication problem associated with fusing edges and lines, where the combination of features produces unpredictable response. The success of the measure function-based hessian tensor benefits from the region information of curved patterns, which include local anisotropy intensity, by quantifying the linearity and coherence quantities. These quantities facilitate a robust detection as they are more sensitive to anisotropic intensity, which makes the proposed function sustainable to low-magnitude eigenvalues, whereas being insensitive to noise regions with constant intensity (isotropy regions).
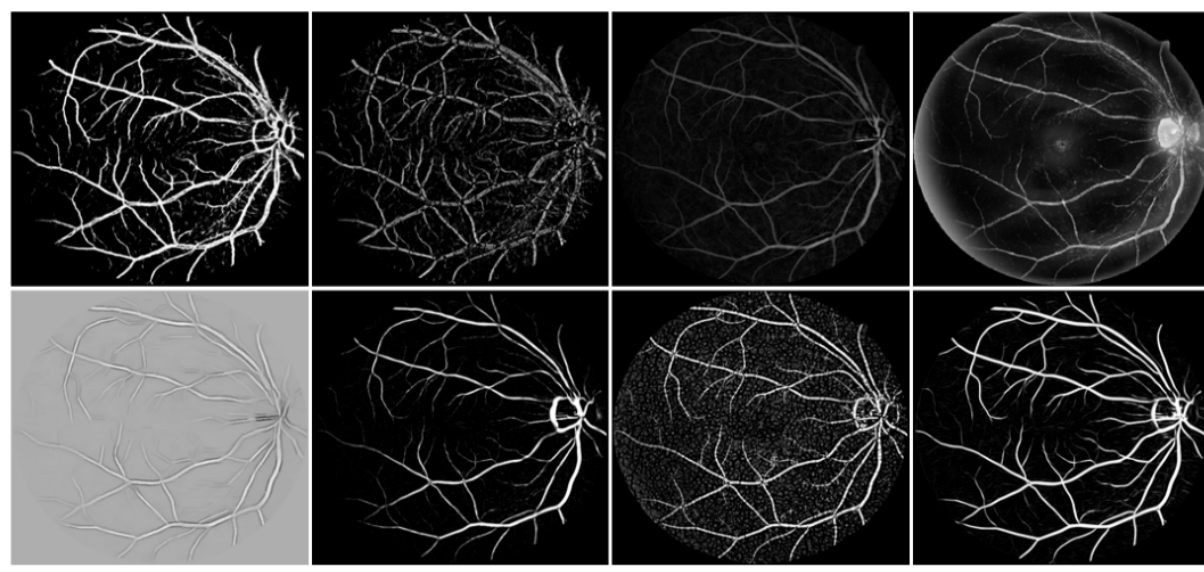

Fig. 5. Detection results of multiple algorithms on a retinal image. First row: PCT-V, PCT-N, MBT, and ISNF, respectively. Second row: MCE, VD, ND, and our method, respectively. 

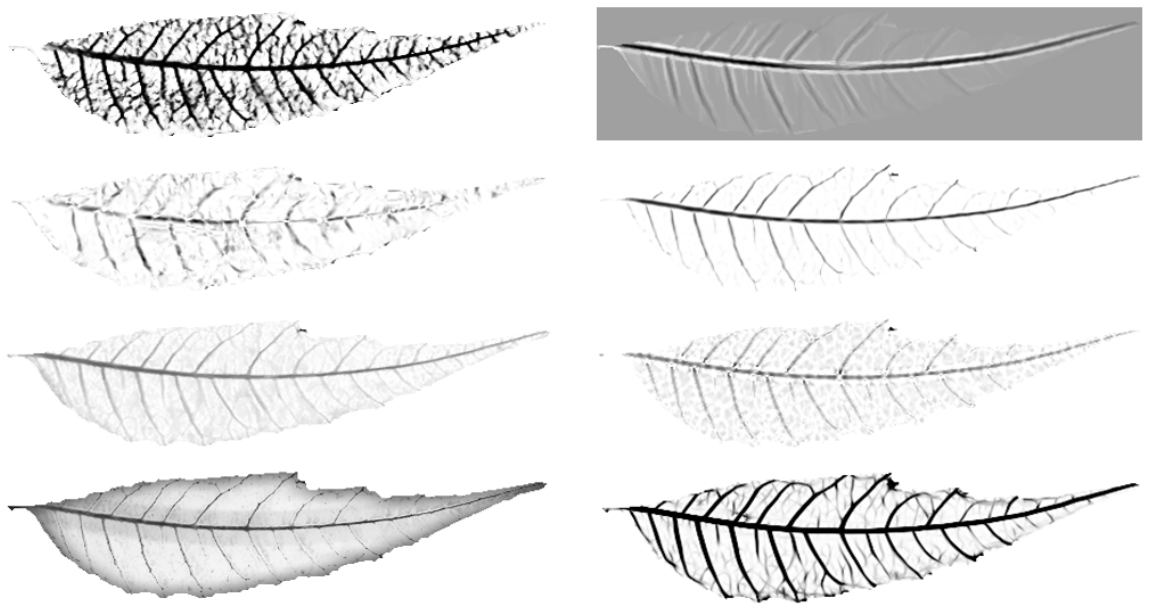

Fig. 6. Detection results of multiple algorithms on a leaf image. First column: PCT-V, PCT-N, MBT, and ISNF, respectively. Second column: MCE, VD, ND, and our method, respectively.

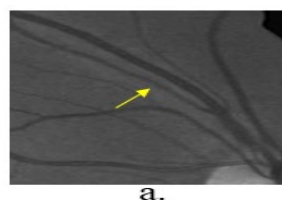

a.

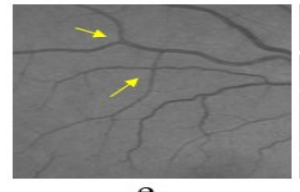

e.

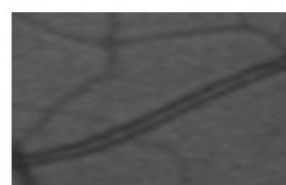

i.

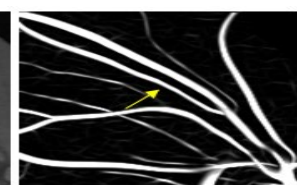

b.

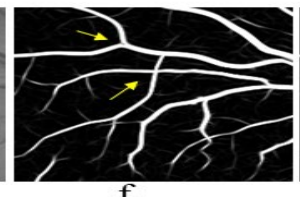

f.

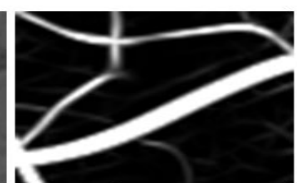

j.

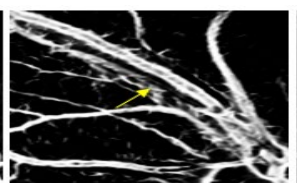

c.

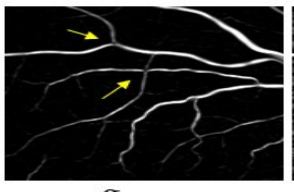

g.

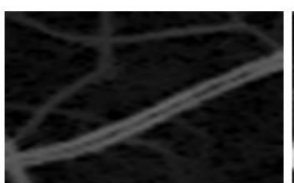

k.

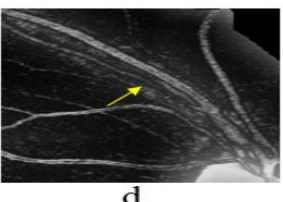

d.
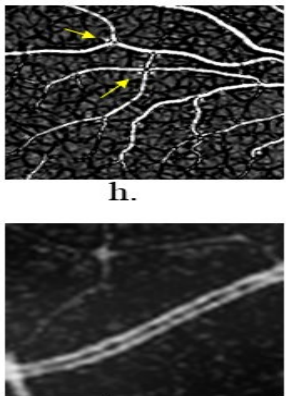

Fig. 7. Detection results of different methods on selective regions. First row: (a) low contrast region, (b) our method, (c) PCT-V, (d) ISNF. Second row: (e) region with bifurcation/crossing, (f) our method, (g) VD, (h) ND. Third row: (i) a vessel with central light reflection, (j) our method, (k) MBT, (l) ISNF.

Moreover, the directional decomposition by the GDQ to curved patterns enables more reliable hessian analysis results, where the eigensystem calculation is facilitated because each directional image includes patterns within the same orientation. The final response is produced by maximizing the function outputs over multiple direc- 
tions to obtain a uniform and strong response across different widths and intensities. Regarding the connectivity of the structure network, the proposed method scheme (decomposition-filtering-recombination) avoids the junction suppression while retaining narrow and low contrast structures.

In conclusion, we introduce a new unsupervised method for the detection of the curvilinear structures in 2D biomedical images. The proposed method utilizes the local phase map estimated by the gaussian derivative quadrature filter to identify line features, and quantifies the geometric information of target structures, by anisotropic linearity and coherence indices, based hessian tensor to propose a novel measure function. The proposed framework has been validated for the extraction of retinal vessel tree and venation pattern, and its results have been compared with state-of-theart benchmark methods to demonstrate its excellent performance. Our detection method is not only able to detect curvilinear tree with a response standing out from the background more conspicuously, but also can suppress noise, retain small and low contrast structures, produce more uniform response, be more robust in the presence of central light reflection and preserve the connectivity of curved pattern. The focus of the future work is to extend the proposed framework to be applied to the vessel extraction from 3D biomedical images and the crack detection in pavement images.

\section{References}

1. Vicas, C., Nedevschi, S.: Detecting Curvilinear Features Using Structure Tensors. IEEE Transactions on Image Processing. 24, 3874-3887 (2015). doi: 10.1109/tip.2015.2447451

2. Obara, B., Fricker, M., Gavaghan, D., Grau, V.: Contrast-Independent Curvilinear Structure Detection in Biomedical Images. IEEE Transactions on Image Processing. 21, 2572-2581 (2012). doi:10.1109/tip.2012.2185938

3. Larese, M., Namías, R., Craviotto, R., Arango, M., Gallo, C., Granitto, P.: Automatic classification of legumes using leaf vein image features. Pattern Recognition. 47, 158168 (2014). doi: 10.1016/j.patcog.2013.06.012

4. Sazak, Ç., Nelson, C., Obara, B.: The multiscale bowler-hat transform for blood vessel enhancement in retinal images. Pattern Recognition. 88, 739-750 (2019). doi: 10.1016/j.patcog.2018.10.011

5. Liu, L., Zhang, D., You, J.: Detecting Wide Lines Using Isotropic Nonlinear Filtering. IEEE Transactions on Image Processing. 16, 1584-1595 (2007). doi:10.1109/tip.2007.894288

6. Zana, F., Klein, J.: Segmentation of vessel-like patterns using mathematical morphology and curvature evaluation. IEEE Transactions on Image Processing. 10, 1010-1019 (2001). doi: 10.1109/83.931095

7. Frangi, A., Niessen, W., Vincken, K., Viergever, M.: Multiscale Vessel Enhancement Filtering. International Conference on Medical Image Computing and ComputerAssisted Intervention. pp. 130-137. Springer (1998). doi: 10.1007/BFb0056195

8. Meijering, E., Jacob, M., Sarria, J., Steiner, P., Hirling, H., Unser, M.: Design and validation of a tool for neurite tracing and analysis in fluorescence microscopy images. Cytometry. 58A, 167-176 (2004). doi:10.1002/cyto.a.20022 
9. Boukerroui, D., Noble, J., Brady, M.: On the Choice of Band-Pass Quadrature Filters. Journal of Mathematical Imaging and Vision. 21, 53-80 (2004). doi:10.1023/b:jmiv.0000026557.50965.09

10. Peeters, T., Rodrigues, P., Vilanova, A., ter Haar Romeny, B.: Analysis of distance/similarity measures for diffusion tensor imaging. In: Laidlaw, D., Weickert, J. (eds.) Visualization and Processing of Tensor Fields. MATHVISUAL, pp. 113-136. Springer, Heidelberg (2009). doi.org/10.1007/978-3-540-88378-4_6

11. Weickert, J.: Coherence-Enhancing Diffusion Filtering. International Journal of Computer Vision. 31, 111-127 (1999). doi: 10.1023/a:1008009714131

12. Challoob, M., Gao, Y.: Retinal Vessel Segmentation Using Matched Filter with Joint Relative Entropy. International Conference on Computer Analysis of Images and Patterns. pp. 228-239. Springer (2017). doi: 10.1007/978-3-319-64689-3_19

13. Marín, D., Aquino, A., Gegundez-Arias, M., Bravo, J.: A new supervised method for blood vessel segmentation in retinal images by using gray-level and moment invariantsbased features. IEEE Transaction on Medical Imaging. 30, 146-158 (2011). doi:10.1109/tmi.2010.2064333

14. You, X., Peng, Q., Yuan, Y., Cheung, Y., Lei, J.: Segmentation of retinal blood vessels using the radial projection and semi-supervised approach. Pattern Recognition. 44, 2314-2324 (2011). doi:10.1016/j.patcog.2011.01.007

15. Soares, J., Leandro, J., Cesar, R., Jelinek, H., Cree, M.: Retinal vessel segmentation using the 2-D Gabor wavelet and supervised classification. IEEE Transaction on Medical Imaging. 25, 1214- 1222 (2006). doi:10.1109/tmi.2006.879967

16. Lázár, I., Hajdu, A.: Segmentation of retinal vessels by means of directional response vector similarity and region growing. Computers in Biology and Medicine. 66, 209-221 (2015). doi: 10.1016/j.compbiomed.2015.09.008

17. Cheng, E., Du, L., Wu, Y., Zhu, Y., Megalooikonomou, V., Ling, H.: Discriminative vessel segmentation in retinal images by fusing context-aware hybrid features. Machine Vision and Applications. 25, 1779-1792 (2014). doi: 10.1007/s00138-014-0638-x

18. Zhao, Y., Wang, X., Wang, X., Shih, F.: Retinal vessels segmentation based on level set and region growing. Pattern Recognition. 47, 2437-2446 (2014). doi: 10.1016/j.patcog.2014.01.006

19. Zhao, Y., Rada, L., Chen, K., Harding, S., Zheng, Y.: Automated Vessel Segmentation Using Infinite Perimeter Active Contour Model with Hybrid Region Information with Application to Retinal Images. IEEE Transactions on Medical Imaging. 34, 1797-1807 (2015). doi: 10.1109/tmi.2015.2409024

20. Lam, B., Gao, Y., Liew, A.: General Retinal Vessel Segmentation Using RegularizationBased Multiconcavity Modeling. IEEE Transactions on Medical Imaging. 29, 13691381 (2010). doi: 10.1109/tmi.2010.2043259

21. Nguyen, U., Bhuiyan, A., Park, L., Ramamohanarao, K.: An effective retinal blood vessel segmentation method using multi-scale line detection. Pattern Recognition. 46, 703715 (2013). doi:10.1016/j.patcog.2012.08.009

22. Fraz, M., Barman, S., Remagnino, P., Hoppe, A., Basit, A., Uyyanonvara, B., Rudnicka, A., Owen, C.: An approach to localize the retinal blood vessels using bit planes and centerline detection. Computer Methods and Programs in Biomedicine. 108, 600-616 (2012). doi: 10.1016/j.cmpb. 2011.08.009

23. Zhang, B., Zhang, L., Zhang, L., Karray, F.: Retinal vessel extraction by matched filter with first-order derivative of Gaussian. Computers in Biology and Medicine. 40, 438445 (2010). doi:10.1016/j. compbiomed.2010.02.008 